
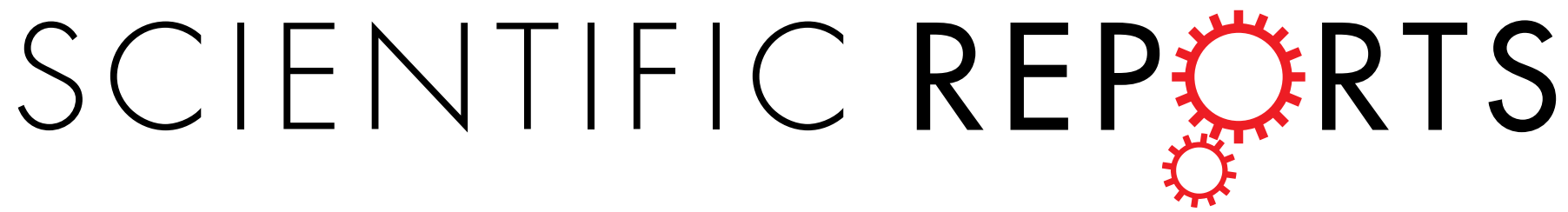

Corrected: Author Correction

Received: 29 November 2018 Accepted: 14 May 2019 Published online: 04 June 2019

\section{Systematic assessment of} prescribed medications and shortterm risk of myocardial infarction - a pharmacopeia-wide association
study from Norway and Sweden

Abhijit Sen ${ }^{1,2}$, loannis Vardaxis ${ }^{3}$, Bo Henry Lindqvist ${ }^{3}$, Ben Michael Brumpton ${ }^{4,5,6}$, Linn Beate Strand $^{1}$, Inger Johanne Bakken (D ${ }^{7}$, Lars Johan Vatten ${ }^{1}$, Pål Richard Romundstad ${ }^{1}$, Rickard Ljung $^{8}$, Kenneth Jay Mukamal ${ }^{9}$ \& Imre Janszky ${ }^{1,10}$

Wholesale, unbiased assessment of Scandinavian electronic health-care databases offer a unique opportunity to reveal potentially important undiscovered drug side effects. We examined the shortterm risk of acute myocardial infarction (AMI) associated with drugs prescribed in Norway or Sweden. We identified 24,584 and 97,068 AMI patients via the patient- and the cause-of-death registers and linked to prescription databases in Norway (2004-2014) and Sweden (2005-2014), respectively. A casecrossover design was used to compare the drugs dispensed 1-7 days before the date of AMI diagnosis with 15-21 days' time -window for all the drug individually while controlling the receipt of other drugs. A BOLASSO approach was used to select drugs that acutely either increase or decrease the apparent risk of AMI. We found 48 drugs to be associated with AMI in both countries. Some antithrombotics, antibiotics, opioid analgesics, adrenergics, proton-pump inhibitors, nitroglycerin, diazepam, metoclopramide, acetylcysteine were associated with higher risk for AMI; whereas angiotensin-IIantagonists, calcium-channel blockers, angiotensin-converting-enzyme inhibitors, serotonin-specific reuptake inhibitors, allopurinol, mometasone, metformin, simvastatin, levothyroxine were inversely associated. The results were generally robust in different sensitivity analyses. This study confirms previous findings for certain drugs. Based on the known effects or indications, some other associations could be anticipated. However, inverse associations of hydroxocobalamin, levothyroxine and mometasone were unexpected and needs further investigation. This pharmacopeia-wide association study demonstrates the feasibility of a systematic, unbiased approach to pharmacological triggers of AMI and other diseases with acute, identifiable onsets.

More than $90 \%$ of all US adults aged 65 years and older use at least one prescription medication ${ }^{1}$, a proportion that continues to increase. Adverse and often unanticipated drug reactions are a major public health challenge. They are estimated to cost around $\$ 30$ billion annually in the US alone ${ }^{2}$.

${ }^{1}$ Department of Public health and Nursing, Faculty of Medicine and Health Sciences, Norwegian University of Science and Technology, 7491, Trondheim, Norway. ${ }^{2}$ Center for Oral Health Services and Research (TkMidt), Trondheim, Norway. ${ }^{3}$ Department of Mathematical Sciences, Norwegian University of Science and Technology, 7491, Trondheim, Norway. ${ }^{4}$ Department of Thoracic Medicine, St. Olav's Hospital, Trondheim University Hospital, Trondheim, Norway. ${ }^{5}$ K.G. Jebsen Centre for Genetic Epidemiology, Department of Public Health and Nursing, Norwegian University of Science and Technology, 7491, Trondheim, Norway. ${ }^{6} \mathrm{MRC}$ Integrative Epidemiology Unit, University of Bristol, Bristol, United Kingdom. ${ }^{7}$ Centre for Fertility and Health (CeFH), Norwegian Institute of Public Health, Oslo, Norway. ${ }^{8}$ Unit of Epidemiology, Institute of Environmental Medicine, Karolinska Institutet, SE 171 77, Solna, Stockholm, Sweden. ${ }^{9}$ Department of Medicine, Beth Israel Deaconess Medical Center, Boston, MA, United States. ${ }^{10}$ Regional Center for Health Care Improvement, St Olav's Hospital, Trondheim, Norway. Correspondence and requests for materials should be addressed to A.S. (email: abhijit.sen@ntnu.no) 


\begin{tabular}{|l|l|l|l|}
\hline Characteristics & Total N (\%) & Sweden N (\%) & Norway N (\%) \\
\hline Total AMI patients* & 121,652 & 97,068 & 24,584 \\
\hline Males & $65,171(53.6)$ & $50,583(52.1)$ & $14,588(59.3)$ \\
\hline Age (in years) & \multicolumn{5}{|l|}{} \\
\hline Below 30 & $65(0.1)$ & $43(0.04)$ & $22(0.1)$ \\
\hline $30-39$ & $428(0.4)$ & $256(0.3)$ & $172(0.7)$ \\
\hline $40-49$ & $2836(2.3)$ & $1785(1.8)$ & $1051(4.3)$ \\
\hline $50-59$ & $9299(7.6)$ & $6369(6.6)$ & $2930(11.9)$ \\
\hline $60-69$ & $20,597(16.9)$ & $15,434(15.9)$ & $5163(21)$ \\
\hline $70-79$ & $30,710(25.2)$ & $24,539(25.3)$ & $6171(25.1)$ \\
\hline $80-89$ & $43,126(35.5)$ & $36,113(37.2)$ & $7013(28.5)$ \\
\hline $90-99$ & $14,419(11,9)$ & $12,381(12.8)$ & $2038(8.3)$ \\
\hline$\geq 100$ & $172(0.1)$ & $148(0.2)$ & $24(0.1)$ \\
\hline
\end{tabular}

Table 1. Characteristics of the study sample. *The number reflects the patients who were hospitalized or died due to acute myocardial infarction both within and outside hospital. In addition, the numbers reflects the patients who dispensed prescribed drugs either in the case-period (1 to 7 days) or control-period (15 to 21 days) before the index date for the diagnosis of their AMI in Norway and Sweden, respectively.

Although drugs undergo several phases of testing prior to approval, pre-approval trials are typically just large enough to detect the expected effect on physiological outcomes. These trials are commonly too small and of too short duration to assess meaningful clinical outcomes ${ }^{3}$. Pre-approval trials also tend to disproportionately exclude women, especially in their reproductive age, patients with comorbidities, individuals in lower socioeconomic strata, children, and elderly individuals, all of which limit generalizability of findings ${ }^{4}$. Thus, an urgent need exists for systematic, generalizable monitoring of important clinical effects even in the post-marketing phase. For example, rofecoxib and sibutramine were both withdrawn from the market due to increased risk for acute myocardial infarction $(\mathrm{AMI})^{5,6}$.

Although many adverse drug reactions can be anticipated on the basis of a drug's physiological effects, the example of the human genome has illustrated how modest the predictive power of variants in established candidate pathways can be. In the case of genomics, this has been addressed by technological improvements that enable wholesale, unbiased assessment of millions of genetic variants on a single outcome -genome-wide association studies (GWAS). These studies have identified dozens of novel pathways across a variety of endpoints. In the case of pharmaceuticals, the advent of registers that track the dispensing of prescription medications enables a comparable approach - the pharmacopoeia-wide association study (PWAS) - to perform unbiased assessment of literally all prescribed medications. Such an approach could offer the potential to identify drugs that unexpectedly increase or decrease risk of serious clinical events with maximal generalizability and statistical power.

To test this approach, we undertook a systematic examination of all potentially existing associations between prescribed drugs and short-term risk for AMI using comprehensive nation-wide data from Sweden and Norway. Given the very strong likelihood of confounding by indication in pharmacoepidemiology, we focused on short-term AMI risk and used case-only methods for self-matching. Because this approach may overestimate the effects of drugs used chronically ${ }^{7}$, it yields estimates that are most reliable for drugs typically taken for short time periods (for acute use).

\section{Results}

Among a total of 121,652 AMI patients, 97,068 were from Sweden (79,882 and 17,186 identified via the patient register and the cause of death register, respectively) and 24,584 were from Norway (20,413 and 4,171 identified via the patient register and the cause of death register, respectively). Characteristics of these patients are presented in Table 1.

A total of 1262 and 1535 distinct pharmaceutical drugs were dispensed for AMI patients in Norway and Sweden, respectively. Out of these, 737 and 1081 unique drugs were dispensed for AMI patients in either the case- or control- period, in Norway and Sweden, respectively. Subsequently, a total of 81 and 112 unique drug types were selected in Norway and Sweden via BOLASSO procedure, respectively. Finally, a total of 48 drugs were selected in common from both countries (Figure 1). Table 2 presents the country-specific and the combined estimates of these commonly selected drugs.

Cardiovascular drugs. Several cardiovascular drugs were associated with higher AMI risk, potentially related to their indications for use. These medications included nitroglycerin and different classes of antithrombotic agents (ticagrelor, clopidogrel, enoxaparin, acetylsalicylic acid). On the other hand, certain angiotensin-II-antagonists (losartan, candesartan), calcium-channel blockers (amlodipine, felodipine), and an angiotensin-converting-enzyme inhibitor (ramipril) were associated with lower risk of AMI.

Antibiotics. Use of penicillins, ciprofloxacin, doxycycline, erythromycin, trimethoprim and nitrofurantoin were associated with higher risk of AMI. In contrast, metronidazole was associated with lower risk of AMI. 

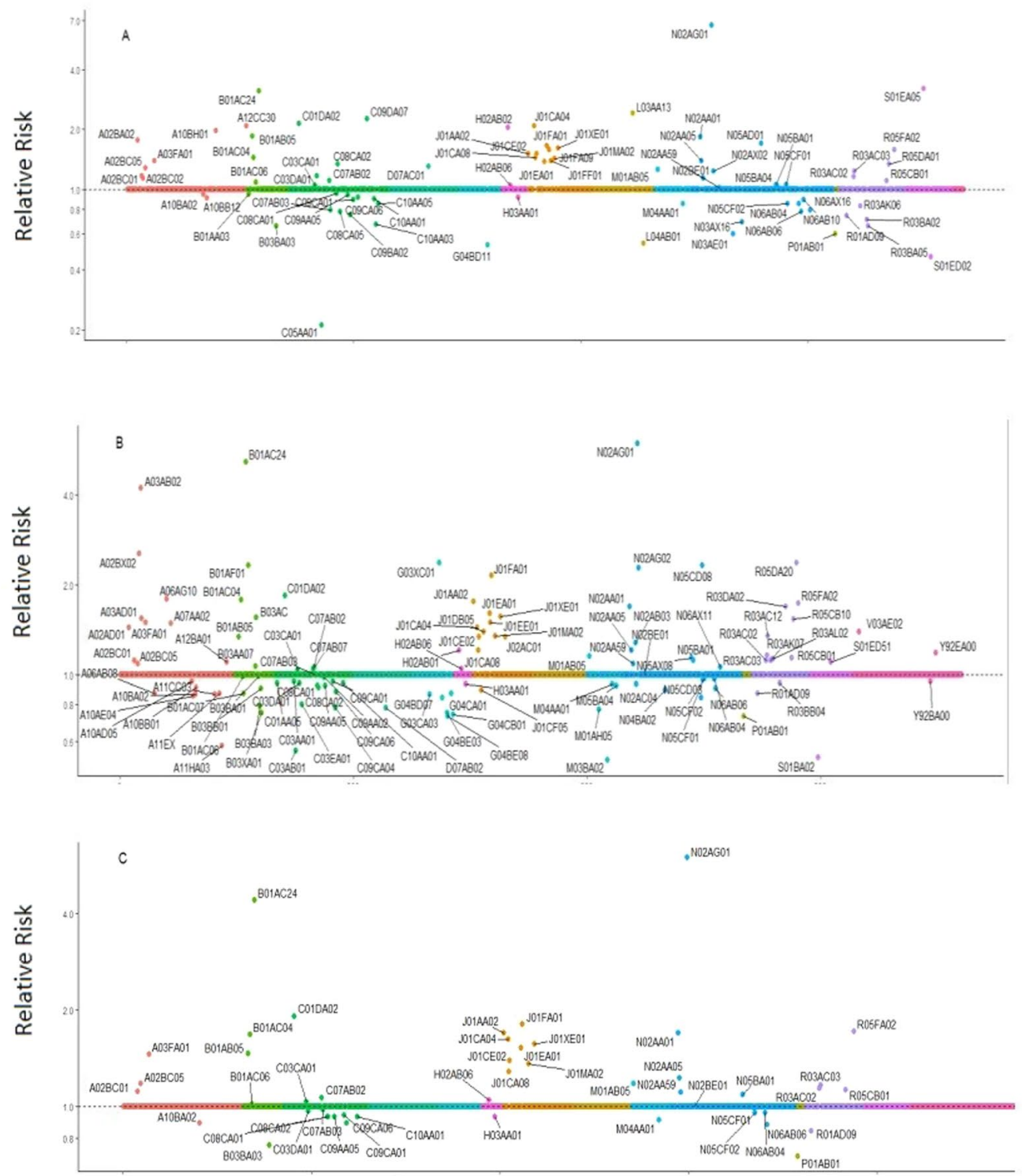

Color by $1^{\text {st }}$ level, anatomical main group

$\begin{array}{llll}\text { - Alimentary tract and metabolism } & \text { - Antiparasitic products } & \text { - Dermatologicals } & \text { - Nervous system - Systemic hormonal preparations, } \\ \text { - Antinfectives for systemic use } & \text { - Blood and blood forming organs } & \text { - Genito-urinary system and sex hormones } & \text { - Respiratory system * Various other } \\ \text { - Antineoplastic and immunomodulating agents } & \text { - Cardiovascular system } & \text { - Musculo-skeletal system } & \text { - Sensory organs }\end{array}$

Figure 1. Pharmacopeia-wide case-crossover analysis of dispensed medication data in relation to risk for acute myocardial infarction. The above plot illustrates (A) 81 unique drug types which were selected in Norway, (B) 112 unique drug types which were selected in Sweden, and (C) 48 drugs which were common hits from both the countries. Y-axis displays relative risk on the log scale, $\mathrm{X}$-axis displays all the prescribed drugs studied grouped according to the Anatomical Therapeutic Chemical (ATC) classification.

Analgesics. Several opioid analgesics were associated with higher risk of AMI. Of these, morphine in combination with antispasmodics was associated with particularly high risk for AMI. Among non-opioid agents, diclofenac was also associated with increased risk for AMI.

Psychoactive medications. Among anxiolytic, antidepressant, and antipsychotic agents, we observed an increased AMI risk in association with the use of diazepam. Two serotonin-specific reuptake inhibitors, citalopram and sertraline, were associated with lower risk for AMI.

Other medications. Specific adrenergic agents (terbutaline, salbutamol), proton pump inhibitors (esomeprazole and omeprazole), metoclopramide and acetylcysteine were associated with higher risk of AMI. On the other hand, allopurinol, mometasone, metformin, simvastatin and levothyroxine were inversely associated with risk of AMI. 


\begin{tabular}{|c|c|c|c|c|c|c|c|c|}
\hline \multirow[b]{2}{*}{ ATC Code $^{s}$} & \multirow[b]{2}{*}{ Drug Names } & \multirow[b]{2}{*}{$\begin{array}{l}\text { Exposed Case- } \\
\text { period only }\end{array}$} & \multirow[b]{2}{*}{$\begin{array}{l}\text { Exposed } \\
\text { Control- } \\
\text { period only }\end{array}$} & \multirow{2}{*}{$\begin{array}{l}\text { Sweden }^{*} \\
\text { RR }(95 \% \mathrm{CI})\end{array}$} & \multirow[b]{2}{*}{$\begin{array}{l}\text { Exposed Case- } \\
\text { period only }\end{array}$} & \multirow[b]{2}{*}{$\begin{array}{l}\text { Exposed } \\
\text { Control- } \\
\text { period only }\end{array}$} & \multirow{2}{*}{\begin{tabular}{|l|} 
Norway $^{\S}$ \\
RR $(95 \% \mathrm{CI})$
\end{tabular}} & \multirow{2}{*}{\begin{tabular}{|l|} 
Total \\
Combined \\
estimates RR \\
$(\mathbf{9 5 \%} \mathrm{CI})$
\end{tabular}} \\
\hline & & & & & & & & \\
\hline \multicolumn{9}{|c|}{ Narcotic analgesics } \\
\hline N02AG01 & $\begin{array}{l}\text { Morphine \& } \\
\text { antispasmodics }\end{array}$ & 256 & 42 & $5.92(4.09-8.57)$ & 28 & 2 & $6.66(2.31-19.36)$ & $6.00(4.23-8.50)$ \\
\hline N02AA01 & Morphine & 2195 & 1515 & $1.69(1.52-1.89)$ & 137 & 67 & $1.84(1.21-2.79)$ & $1.70(1.53-1.89)$ \\
\hline N02AA05 & Oxycodone & 2659 & 2337 & $1.21(1.10-1.34)$ & 252 & 191 & $1.40(1.07-1.84)$ & $1.23(1.12-1.35)$ \\
\hline N02AA59 & Codeine, combinations & 1526 & 1391 & $1.09(1.00-1.20)$ & 1386 & 1202 & $1.14(1.03-1.25)$ & $1.11(1.04-1.19)$ \\
\hline R05FA02 & $\begin{array}{l}\text { Opium derivatives \& } \\
\text { expectorant }\end{array}$ & 828 & 438 & $1.73(1.52-1.97)$ & 71 & 35 & $1.59(1.00-2.54)$ & $1.72(1.52-1.95)$ \\
\hline \multicolumn{9}{|c|}{ Non-narcotic analgesic } \\
\hline N02BE01 & Paracetemol & 14481 & 14036 & $1.01(0.75-1.05)$ & 1412 & 1290 & $1.02(0.93-1.12)$ & $1.01(0.98-1.05)$ \\
\hline \multicolumn{9}{|c|}{ Anti-inflammatory drug } \\
\hline M01AB05 & Diclofenac & 1180 & 1001 & $1.15(1.04-1.28)$ & 504 & 387 & $1.26(1.07-1.47)$ & $1.18(1.08-1.29)$ \\
\hline \multicolumn{9}{|l|}{ Antibiotics } \\
\hline J01FA01 & Erythromycin & 90 & 36 & $2.14(1.39-3.30)$ & 156 & 89 & $1.66(1.22-2.24)$ & $1.81(1.41-2.32)$ \\
\hline J01AA02 & Doxycycline & 1095 & 591 & $1.76(1.58-1.96)$ & 324 & 213 & $1.51(1.25-1.83)$ & $1.70(1.54-1.86)$ \\
\hline J01CA04 & Amoxicillin & 575 & 353 & $1.44(1.24-1.66)$ & 327 & 168 & $2.09(1.69-2.58)$ & $1.62(1.44-1.83)$ \\
\hline J01XE01 & Nitrofurantoin & 365 & 249 & $1.56(1.29-1.89)$ & 105 & 66 & $1.62(1.12-2.33)$ & $1.57(1.33-1.86)$ \\
\hline J01EA01 & Trimethoprim & 402 & 281 & $1.60(1.33-1.92)$ & 149 & 114 & $1.38(1.03-1.84)$ & $1.53(1.31-1.79)$ \\
\hline J01CE02 & Phenoxymethylpenicillin & 847 & 595 & $1.34(1.20-1.50)$ & 423 & 277 & $1.51(1.29-1.77)$ & $1.39(1.27-1.53)$ \\
\hline J01MA02 & Ciproflolaxin & 830 & 630 & $1.35(1.19-1.52)$ & 194 & 134 & $1.43(1.10-1.86)$ & $1.36(1.22-1.52)$ \\
\hline J01CA08 & Pivmecillinam & 535 & 422 & $1.21(1.05-1.41)$ & 309 & 211 & $1.45(1.19-1.78)$ & $1.29(1.14-1.45)$ \\
\hline \multicolumn{9}{|c|}{ Antiprotozoal drug } \\
\hline P01AB01 & Metronidazole & 169 & 185 & $0.73(0.57-0.93)$ & 51 & 51 & $0.60(0.38-0.94)$ & $0.70(0.56-0.87)$ \\
\hline \multicolumn{9}{|c|}{ Antithrombotic agents } \\
\hline B01AC24 & Ticagrelor & 96 & 14 & $5.14(2.85-9.26)$ & 27 & 6 & $3.12(1.27-7.67)$ & $4.42(2.70-7.24)$ \\
\hline B01AC04 & Clopidogrel & 1290 & 852 & $1.78(1.57-2.01)$ & 391 & 251 & $1.44(1.19-1.76)$ & $1.68(1.51-1.86)$ \\
\hline $\mathrm{B} 01 \mathrm{AB} 05$ & Enoxaparin & 126 & 93 & $1.34(0.98-1.83)$ & 78 & 43 & $1.85(1.14-3.00)$ & $1.47(1.13-1.92)$ \\
\hline B01AC06 & Acetylsalicylic acid & 15489 & 15240 & $0.99(0.95-1.03)$ & 3141 & 2803 & $1.09(1.02-1.17)$ & $1.02(0.98-1.05)$ \\
\hline \multicolumn{9}{|c|}{ Antiadrenergic agents } \\
\hline R03AC03 & Terbutaline & 968 & 787 & $1.16(1.04-1.30)$ & 92 & 77 & $1.24(0.89-1.73)$ & $1.17(1.05-1.30)$ \\
\hline R03AC02 & Salbutamol & 618 & 526 & $1.12(0.98-1.28)$ & 653 & 532 & $1.16(1.00-1.34)$ & $1.14(1.03-1.26)$ \\
\hline Proton pump & inhibitors & & & & & & & \\
\hline A02BC05 & Esomeprazol & 871 & 802 & $1.09(0.96-1.25)$ & 725 & 588 & $1.29(1.13-1.48)$ & $1.18(1.08-1.30)$ \\
\hline $\mathrm{A} 02 \mathrm{BC} 01$ & Omeprazole & 8870 & 8261 & $1.12(1.07-1.18)$ & 238 & 213 & $1.17(0.94-1.45)$ & $1.12(1.07-1.18)$ \\
\hline Vasodilator & & & & & & & & \\
\hline C01DA02 & Glyceral trinitrate & 5020 & 2949 & $1.84(1.74-1.94)$ & 1758 & 864 & $2.15(1.96-2.36)$ & $1.92(1.83-2.01)$ \\
\hline Prokinetic $d r$ & & & & & & & & \\
\hline A03FA01 & Metoclopramide & 709 & 503 & $1.49(1.28-1.73)$ & 335 & 220 & $1.40(1.12-1.75)$ & $1.46(1.29-1.66)$ \\
\hline Expectorant & & & & & & & & \\
\hline R05CB01 & Acetylcysteine & 1677 & 1375 & $1.14(1.05-1.24)$ & 519 & 394 & $1.11(0.96-1.30)$ & $1.13(1.05-1.22)$ \\
\hline Anxiolytic & & & & & & & & \\
\hline N05BA01 & Diazepam & 1165 & 957 & $1.12(0.99-1.26)$ & 664 & 575 & $1.06(0.93-1.22)$ & $1.09(1.00-1.20)$ \\
\hline Angiotension & II antagonists & & & & & & & \\
\hline C09CA01 & Losartan & 1728 & 1738 & $0.95(0.86-1.05)$ & 292 & 310 & $0.89(0.73-1.08)$ & $0.94(0.86-1.02)$ \\
\hline C09CA06 & Candesartan & 1363 & 1448 & $0.88(0.79-0.98)$ & 360 & 387 & $0.92(0.77-1.09)$ & $0.89(0.81-0.98)$ \\
\hline Antidepressa & ints & & & & & & & \\
\hline N06AB04 & Citalopram & 5548 & 5513 & $0.97(0.90-1.05)$ & 170 & 182 & $0.85(0.66-1.11)$ & $0.96(0.89-1.03)$ \\
\hline N06AB06 & Sertraline & 1358 & 1360 & $0.90(0.78-1.04)$ & 90 & 98 & $0.78(0.53-1.13)$ & $0.88(0.77-1.01)$ \\
\hline ACE inhibito & & & & & & & & \\
\hline C09AA05 & Ramipril & 1870 & 1855 & $0.92(0.83-1.02)$ & 709 & 683 & $0.94(0.81-1.08)$ & $0.93(0.85-1.01)$ \\
\hline Antigout age & & & & & & & & \\
\hline M04AA01 & Allopurinol & 1897 & 1952 & $0.93(0.83-1.03)$ & 351 & 394 & $0.85(0.70-1.04)$ & $0.91(0.83-1.00)$ \\
\hline Antidiabetic & agent & & & & & & & \\
\hline A10BA02 & Metformin & 2659 & 2896 & $0.86(0.79-0.94)$ & 693 & 695 & $0.95(0.83-1.09)$ & $0.89(0.82-0.95)$ \\
\hline Beta-blockin & gagents & & & & & & & \\
\hline C07AB02 & Metoprolol & 8861 & 8437 & $1.05(1.00-1.11)$ & 2579 & 2289 & $1.11(1.02-1.20)$ & $1.07(1.02-1.12)$ \\
\hline Continued & & & & & & & & \\
\hline
\end{tabular}




\begin{tabular}{|c|c|c|c|c|c|c|c|c|}
\hline \multirow[b]{2}{*}{ ATC Code $^{s}$} & \multirow[b]{2}{*}{ Drug Names } & \multirow[b]{2}{*}{$\begin{array}{l}\text { Exposed Case- } \\
\text { period only }\end{array}$} & \multirow[b]{2}{*}{$\begin{array}{l}\text { Exposed } \\
\text { Control- } \\
\text { period only }\end{array}$} & \multirow{2}{*}{\begin{tabular}{|l|} 
Sweden $^{*}$ \\
RR (95\% CI)
\end{tabular}} & \multirow[b]{2}{*}{$\begin{array}{l}\text { Exposed Case- } \\
\text { period only }\end{array}$} & \multirow[b]{2}{*}{$\begin{array}{l}\text { Exposed } \\
\text { Control- } \\
\text { period only }\end{array}$} & \multirow{2}{*}{\begin{tabular}{|l|} 
Norway $^{\S}$ \\
RR (95\% CI)
\end{tabular}} & \multirow{2}{*}{\begin{tabular}{|l|} 
Total \\
Combined \\
estimates RR \\
$(\mathbf{9 5 \%}$ CI)
\end{tabular}} \\
\hline & & & & & & & & \\
\hline $\mathrm{C} 07 \mathrm{AB} 03$ & Atenolol & 2836 & 2823 & $1.01(0.93-1.09)$ & 252 & 285 & $0.79(0.65-0.96)$ & $0.98(0.91-1.05)$ \\
\hline \multicolumn{9}{|c|}{ Calcium-channel blockers } \\
\hline C08CA01 & Amlodipine & 2512 & 2544 & $0.92(0.85-1.00)$ & 706 & 718 & $0.96(0.85-1.09)$ & $0.93(0.87-1.00)$ \\
\hline C08CA02 & Felodipine & 3529 & 3617 & $0.91(0.85-0.98)$ & 112 & 93 & $1.34(0.96-1.87)$ & $0.93(0.86-0.99)$ \\
\hline \multicolumn{9}{|c|}{ Decongestant } \\
\hline R01AD09 & Mometasone & 326 & 338 & $0.87(0.73-1.03)$ & 83 & 100 & $0.74(0.54-1.03)$ & $0.84(0.72-0.98)$ \\
\hline \multicolumn{9}{|l|}{ Diuretics } \\
\hline C03CA01 & Furosemide & 14307 & 13807 & $1.04(0.99-1.09)$ & 1317 & 1201 & $1.05(0.95-1.17)$ & $1.04(1.00-1.09)$ \\
\hline C03DA01 & Spironolactone & 2716 & 2674 & $0.94(0.86-1.03)$ & 258 & 219 & $1.17(0.92-1.48)$ & $0.97(0.89-1.05)$ \\
\hline \multicolumn{9}{|c|}{ Glucocorticoid } \\
\hline H02AB06 & Prednisolone & 3692 & 3486 & $1.05(0.97-1.13)$ & 963 & 823 & $1.05(0.94-1.17)$ & $1.05(0.99-1.12)$ \\
\hline \multicolumn{9}{|c|}{ Hypnotics and sedatives } \\
\hline N05CF01 & Zopiclone & 5999 & 6024 & $0.96(0.90-1.02)$ & 1618 & 1460 & $1.06(0.97-1.17)$ & $0.99(0.94-1.04)$ \\
\hline N05CF02 & Zolpidem & 2555 & 2564 & $0.97(0.89-1.05)$ & 175 & 191 & $0.85(0.67-1.07)$ & $0.96(0.88-1.03)$ \\
\hline \multicolumn{9}{|c|}{ Lipid modifying agent } \\
\hline C10AA01 & Simvastatin & 5611 & 5672 & $0.94(0.88-0.99)$ & 1806 & 1748 & $0.90(0.82-0.98)$ & $0.93(0.88-0.97)$ \\
\hline \multicolumn{9}{|c|}{ Antithyroid agent } \\
\hline H03AA01 & Levothyroxine sodium & 4843 & 4863 & $0.93(0.86-1.01)$ & 629 & 648 & $0.92(0.80-1.06)$ & $0.93(0.87-1.00)$ \\
\hline \multicolumn{9}{|c|}{ Antianemic agent } \\
\hline B03BA03 & Hydroxocobalamin & 125 & 155 & $0.79(0.61-1.01)$ & 49 & 63 & $0.66(0.43-1.02)$ & $0.76(0.61-0.94)$ \\
\hline
\end{tabular}

Table 2. Relative risks of acute myocardial infarction within 7 days after the drug was dispensed, selected by BOLASSO approach ${ }^{\dagger}$ in both countries. *Case crossover analysis, case period (1 to 7 days) and control period (15 to 21 days) before the index-date for the diagnosis of AMI. 'In BOLASSO, the effect of each selected drugs is controlled for the effect of other selected drugs. ${ }^{\ddagger}$ Data from the Swedish Prescribed Drug Register, 2005 to 2014. ${ }^{\S}$ Data from the Norwegian Prescription Database, 2004 to 2014. "Combined estimates of Norwegian and

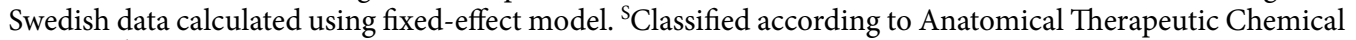
(ATC), $5^{\text {th }}$ level.

In online supplementary material, Tables S1 and S2, we present all drugs selected by BOLASSO in either Norway or Sweden, respectively. Apart from the results of the BOLASSO, we also present the univariable, non-penalized estimates for the selected drugs in these tables.

Sensitivity analysis. In online Supplementary Table S3, we present the results of our analyses where we extended the case-, control- and wash-out periods from 7 days to 14 days. Point estimates were generally similar to those in our main analyses. However, these analyses selected slightly more drugs. Some additional cardiovascular drugs (vasodilator isosorbide mononitrate, diuretic bendroflumethiazide, beta-blocker bisoprolol) were associated with higher AMI risk; whereas others (angiotensin II antagonist valsartan, calcium channel blocker amlodipine, pravastatin, atorvastatin and diuretic spironolactone) were associated with lower risk. Some additional antibiotics (clindamycin and nystatin), opioid analgesics (ketobemidone in combination with antispasmodics, fentanyl, tramadol), the anti-cancer drug capecitabine, the anxiolytic oxazepam and the antidepressant mirtazapine were also associated with increased risk. For norethisterone and estrogen, tolterodine, hydrocortisone, budesonide, tiotropium bromide, salmeterol and fluticasone, we observed lower risk in the extended analyses.

In online Supplementary Table S4, we present analyses restricted to those AMI cases diagnosed in hospital. The results were largely similar to the main analyses, but BOLASSO selected fewer drugs, possibly due to the lower statistical power.

In online Supplementary Tables S5 and S6, we present sub-group analyses by age (dichotomized at 80 years) for 48 drugs selected via BOLASSO approach in both countries. Relative risks were generally comparable between the two age groups.

\section{Discussion}

In this pharmacopeia-wide association study, we investigated all possible associations between pharmaceutical drugs requiring a prescription and short-term risk for AMI using nationwide data from two countries, Norway and Sweden. We identified 48 drugs that were either associated with increase or decrease in short-term risk for AMI in both countries.

Our approach had similarities with genome-wide association studies, but also some important differences that are reflected in our analytic strategy. Bonferroni or similar adjustments are widely used in genome-wide association studies to handle the problem of multiple comparisons. These methods adjust the $p$ value or the significance level based on the number of tests performed and decrease the occurrence of false positive findings, albeit at the 
expense of increasing the occurrence of false negative findings. The Bonferroni method also assumes that associations are essentially random and tests a joint null hypothesis that no exposures are associated with the outcome. This might be justifiable for genome-wide association studies, where, at most, only a handful of true associations are likely out of millions of tested candidates. However, such a joint null hypothesis makes less sense in our study, because many medications clearly do influence AMI risk ${ }^{8}$. Finally, and most importantly, Bonferroni and related methods can increase the rate of true positive findings but cannot improve estimation. On the contrary, estimates for effects selected based on Bonferroni adjusted significance are likely to be substantially inflated. In contrast, penalized regression can greatly improve the accuracy of estimation over conventional methods in the setting of multiple comparisons ${ }^{9,10}$.

It may be useful to consider the medications that we found to be associated with AMI risk in several categories, based upon standard epidemiological principles. In contrast to GWAS, PWAS examines medications that vary frequently in time and bear specific indications, and hence are prone to confounding and other issues for which appropriate epidemiological cautions are necessary. First are cases in which reverse causation may be at play. This includes drugs specifically used to treat accelerating symptoms of myocardial ischemia such as analgesics, nitroglycerin or metoprolol, but also drugs used to treat conditions that produce chest pain or other ischemic symptoms, such as gastroesophageal reflux, anxiety attacks or respiratory conditions. This latter could explain the observed associations for proton pump inhibitors, antiadrenergic agents, and anxiolytics.

One approach to address reverse causation is comparison within a single drug class, where members usually have similar if not identical indications. Large differences between the observed associations for drugs within the same class might indicate true differences in cardiovascular effects. For example, morphine in combination with antispasmodics had a much stronger association with short-term risk of AMI than other opioids, suggesting that this combination could be a strong candidate for further in-depth studies that may eventually result in regulatory changes. Also, ticagrelor had a considerably stronger association with AMI risk than other antithrombotic agents, although this may reflect its narrower range of use, namely that is typically prescribed in the acute phases before and after AMI when the risk of recurrence is highest.

Second are cases of probable confounding. This particularly affects drugs used to treat conditions that themselves increase risk of AMI, such as antibiotics to treat infections and drugs to treat inflammatory or acute painful conditions that may increase risk similarly. Although these associations are unlikely to be causal, PWAS offers the particular advantage that it may, by proxy, identify important physiological pathways leading to AMI. Although infection has been associated with triggering of AMI in previous studies ${ }^{11}$, our much larger study provides a richer source of evidence for this hypothesis. Much like reverse causation, comparison within class, even for confounders, may yield important insights, and our results suggest that metronidazole may acutely lower risk of AMI. Suggestively, this may reflect the disulfiram-like activity of metronidazole, given the association of acute alcohol intake with $\mathrm{AMI}^{12}$ and the absence of other commonly-used drugs that inhibit aldehyde dehydrogenase activity. Alternatively, animal and in vitro studies have suggested that pathogens such as helminths might protect against atherosclerosis via activation of the Th2 pathway ${ }^{13,14}$. This could imply that the association of metronidazole with a lower risk of AMI might reflect a cardioprotective effect of an underlying parasitic infection. However, given the broad range of indications for metronidazole, further study of this possibility is needed.

Third are true associations in which drugs may causally influence AMI risk. This group includes drugs that plausibly increases or decreases risk themselves and, equally, in which withdrawal of an existing medication increases risk. The latter mechanism is the most plausible explanation for the inverse association with AMI for statins, calcium-channel blockers, angiotensin II antagonists and angiotensin-converting-enzyme inhibitors. Our results suggest that clinicians should exercise caution in withdrawing these medications to avoid rebound increases in risk. The inverse associations between AMI risk and some antidepressants, allopurinol and metformin were also expected based on prior epidemiological studies ${ }^{15-18}$ and the known pathophysiological effects of these drugs. For example, SSRIs possess acute anti-platelet effects ${ }^{19}$, allopurinol seems to reduce oxidative stress, to prevent plaque instability and to have anti-ischemic properties ${ }^{20}$ and metformin may improve endothelial function ${ }^{21}$. However, our results provide large-scale evidence of associations that extend to triggering of clinical events.

Lastly, PWAS has the potential to identify new, unexpected agents, where the mechanism of action is uncertain but that clearly require further study. The inverse associations between AMI risk and use of hydroxocobalamin, levothyroxine and mometasone are examples for such unexpected and unexplained findings in the present study. We are not aware of earlier studies suggesting acute cardioprotection for these drugs; neither can these associations be readily explained by the indication nor by the known effects of these drugs. It is possible that they are markers of clinical stability and only prescribed when patients are at particularly low risk, but further clinical detail would be needed to explore this possibility.

To the best of our knowledge, only two studies, one from the United States ${ }^{22}$ and another from Europe ${ }^{23}$ have adopted somewhat similar approaches and investigated the association between drugs and AMI in a hypothesis-free manner. In contrast to our study, the data from these prior studies were not nationwide, but relied on databases of administrative claims ${ }^{24}$, and the endpoint of AMI was not based on a clinical diagnosis in a hospital. None of the studies applied a case-crossover design. Furthermore, these studies used Bonferroni adjustment for multiple comparisons. Thus, the effect sizes were not comparable with those in our study. However, overall, the group of drugs selected as being associated with AMI risk in these two earlier studies were generally similar to those in our study, further supporting the value of this approach as a reproducible method potentially appropriate for standardization, automation, and continuous monitoring of sentinel events within national health systems. In this regard, our approach fits within the rubric of adaptive biomedical innovation, which seeks to apply real-time, large-scale, rapid-cycle clinical data to the process of approving, monitoring, and targeting new pharmaceuticals, devices, and other forms of therapy ${ }^{25}$. 
Strength and limitations. We conducted a nation-wide study in two countries where health care systems are universal and equally accessible to virtually all the residents. Reporting is mandatory in the patient registers, cause of death registries and prescription databases in both Norway and Sweden. Therefore, our study is not subject to bias due to self-selection or to recall bias. Validation studies confirm that the quality of information in the Norwegian and Swedish Patient Registers is very high on $\mathrm{AMI}^{26,27}$. Furthermore, the prescription databases in both countries are fully digitalized, and hence little chance of misclassification of drug retrieval exists. Due to the case-crossover design, our results are unlikely to be confounded by stable patient characteristics, chronic conditions or lifestyle related factors predisposing to AMI and influencing medication use ${ }^{28}$, although they are prone to confounding by characteristics that fluctuate within individuals over days-to-weeks.

Apart from these strengths, our study also has important limitations. Although we used a BOLASSO to minimize the problem of multiple comparisons and relied on nation-wide data from two countries, but nonetheless we cannot rule out the possibility that some associations are due to chance and further studies are needed to confirm the findings from this large-scale screening. This limitation is common to all broad-based, agnostic screening approaches. Similar to GWAS, our study should be regarded as a first step in monitoring the pharmacopoeia, with direct clinical impact occurring later. For example, the first GWAS on age-related macular degeneration was conducted in $2005^{29}$, and it eventually led to promising therapies for age-related macular degeneration in $2016^{30}$.

Given the exploratory nature of our work, we analyzed all drugs in a uniform way and consequently the hypothesized case-, control- and wash-out periods might not be optimal for several drugs. It is important to recognize that such uncertainties lead to a bias towards null in a case-crossover study ${ }^{28,31}$. Thus, under- but not overestimation of effects is likely in case of some drugs due to the suboptimal length of time windows. It is important to emphasize, however, that when we extended the case-, control- and wash-out periods in our sensitivity analyses, we observed generally similar results. Also, case-crossover studies are prone to the so-called 'persistent user bias ${ }^{77}$ which might lead to an upward bias of the estimates in case of chronically-used drugs. As this study lacks data to distinct between medications for acute use and chronic use. Thus, these results must be interpret cautiously on clinical grounds; particularly those prescribed drugs that are used chronically. However, it cannot explain the novel findings in this study, such as the observed protective effect of hydroxocobalamin, levothyroxine and mometasone, nor to differences within class. Also, given the register-based nature of our study, we do not have additional information on patient characteristics such as cardiovascular risk factors or previous cardiac events, although our use of the case-crossover design precludes confounding by stable personal characteristics.

Finally, Norwegian Prescription Database and Swedish Prescribed Drug Register contain complete and valid information on dispensed drugs, but they do not describe the actual date of self-administration. This most probably led to a non-differential misclassification and a bias toward null findings. In this regard, claims-based datasets may have potential advantages for extending and refining this approach.

In summary, this pharmacopeia-wide association study demonstrates the feasibility and results of a systematic, unbiased approach to pharmacological triggers of AMI. Our results also highlight the value of rigorous epidemiological consideration of observed findings. This approach also offers the possibility of routine, rigorous monitoring of the worldwide pharmacopeia for agents that trigger, or prevent the triggering of, serious acute health events.

\section{Methods}

Study design. We applied a case-crossover design ${ }^{28,31}$, which includes only cases of a disease and applies self-matching by comparing exposure before the disease onset with disease-free time in the past as control information. This design is a special case of the conventional matched case-control design, and it has common features with cross-over trials. A primary advantage of the case-crossover design is that stable within-person characteristics cannot confound observed associations, although time-varying features can. Today, it is largely considered a standard method to study acute or triggering effects of transient exposures on serious outcomes such as $\mathrm{AMI}^{32-34}$.

Ascertainment of AMI. We used the Norwegian Patient Registry, the Swedish National Patient Registry, and cause of death registries in Norway and Sweden to identify AMI cases ${ }^{35,36}$. Validation studies show that the quality of information on AMI in these registers, especially in the Norwegian Patient Registry and the Swedish National Patient Register is very high ${ }^{26,27}$. In Norway, all patients with primary ICD-10 hospital discharge diagnoses of I2 1 or I22 from 1 January 2008 to 31 December 2014 were included, as were individuals with a comparable cause of death from 1 January 2004 to 31 December 2014. In Sweden, the corresponding dates for both the hospital diagnosis and cause of death were between $1^{\text {st }}$ November 2005 and $31^{\text {st }}$ December 2014. For each individual, only the first registered episode of AMI was included in the analyses.

Prescribed medications. We assessed the risk of AMI associated with each and every drug prescribed to AMI patients. Data on dispensed medications prior to the occurrence of AMI were abstracted from the nation-wide registration of dispensed drugs in Norway and Sweden, respectively. The Norwegian Prescription Database was established in $2004^{37}$. All Norwegian pharmacies are required to supply information from prescriptions including type and dosage of the drug and date of drug dispense. Sweden has also established a similar register, the Swedish Prescribed Drug Register in $2005^{38}$. A personal identifier is attached to these data that makes it possible to link the information on drug use to other health related registers existing in these countries. The prescription databases do not include information on drugs purchased over-the-counter, or given to institutionalized patients in nursing homes or hospitals. In Norway, it was possible to exclude participants who, at the time of AMI, were institutionalized and for whom registration of dispensed medications was not available. In Sweden, in the absence of this information, we included only those patients who had at least one drug prescribed during one year before the occurrence of AMI. 
Statistical analyses. For each patient, the occurrence of drug dispensing within 1 to 7 days before the date of diagnosis of AMI (case period) was compared to a time window of 15 to 21 days before the AMI diagnosis (control period) for each drug individually. To minimize the carryover effects of drugs, we included a one-week wash-out period between the case- and the control-periods. We calculated odds ratios together with $95 \%$ confidence intervals, comparing the odds of drug dispensed in the case period to that in the control period using conditional logistic regression.

We assessed all prescribed medications in relation to AMI risk. Because our aim was to identify drugs with a real effect and to assess the size of these effects, we opted not to use Bonferroni adjustment and similar conventional methods to address the problem of multiple comparisons inherent to such settings, as they fail to estimate the size of these associations correctly ${ }^{10}$. Instead, we applied a version of the least absolute shrinkage and selection operator (LASSO) regression analysis ${ }^{9,10,39-41}$ called BOLASSO (bootstrap-enhanced least absolute shrinkage operator $)^{42}$. With the BOLASSO, a number of bootstrap samples are drawn from the dataset, where each bootstrap sample is generated by sampling $\mathrm{N}$ pairs ( $\mathrm{N}$ is the total number of drugs in the dataset) with replacement. Here, we have drawn 1000 bootstrap samples. Of note, confidence intervals generated via BOLASSO approach are not optimal, because each bootstrap sample is estimated on different penalty parameters. Therefore, some of the drugs selected by this approach may include one in their confidence interval. In online supplementary material, Appendix A, we present in detail the background of the method and how we implemented BOLASSO in conditional logistic regression models for case-crossover data.

We conducted separate analyses for Norwegian and Swedish data. We presented both country-specific and combined estimates for drugs selected by BOLASSO from both countries. In BOLASSO, the effect of each selected drug is controlled for the effects of all other selected drugs. The combined estimates were calculated using fixed-effect models ${ }^{43}$.

We performed sensitivity analyses to examine the robustness of our results. Firstly, we extended the case-, control- and wash-out periods from 7 days to 14 days (case period $=1$ to 14 days; control period $=29$ to 42 days) and repeated all analyses. Secondly, because a diagnosis of AMI is likely to be more reliable when given at a hospital than when provided as a cause of death, we also repeated our main analyses when we excluded patients who died outside of hospital. Lastly, we performed subgroup analyses by age (dichotomized at 80 years) on drugs selected by BOLASSO approach in both the countries. Separate analyses were performed for Norwegian and Swedish data.

All statistical analyses were performed using R (version 3.2.3; R foundation for Statistical Computing, Vienna, Austria) and Stata/IC 14 (Stata Corp, College Station, Texas, USA).

Ethical approval and informed consent. Informed consent for study participation was obtained by the national registers from the respective country. The data for the patients from the respective registers was send to us anonymously. Thus, in our present research, no patients were involved in setting the research question or the outcome measures, nor were they involved in developing plans for recruitment, design, or implementation of the study. No patients were asked to advise on interpretation or writing up of results.

The studies were approved by the Regional Committees for Medical and Health Research Ethics (REC) in Central Norway and Regional Ethical Review Board in Sweden. In addition, Norwegian data was also approved by Norwegian Data Protection Authority (Datatilsynet). All methods were performed in accordance with the relevant guidelines and regulations by the respective ethical committees from both Norway and Sweden.

\section{Data Availability}

The data that support the findings of this study are available from the Norwegian Patient Registry, Norwegian Prescription database, Norwegian Cause of Death Registry, and Swedish National Patient Registry, Swedish Prescribed Drug Registry, Swedish Cause of Death Registry but restrictions apply to the availability of these data, which were used under license for the current study, and so are not publicly available. Data are however available from the corresponding authors upon reasonable request and with permission of respective national registry in Norway and Sweden.

\section{References}

1. Center for Disease Control and Prevention, https://www.cdc.gov/nchs/data/hus/hus16.pdf\#079.

2. Sultana, J., Cutroneo, P. \& Trifiro, G. Clinical and economic burden of adverse drug reactions. J Pharmacol Pharmacother 4, S73-77, https://doi.org/10.4103/0976-500X.120957 (2013).

3. Sorensen, H. T., Lash, T. L. \& Rothman, K. J. Beyond randomized controlled trials: a critical comparison of trials with nonrandomized studies. Hepatology 44, 1075-1082, https://doi.org/10.1002/hep.21404 (2006).

4. Van Spall, H. G., Toren, A., Kiss, A. \& Fowler, R. A. Eligibility criteria of randomized controlled trials published in high-impact general medical journals: a systematic sampling review. JAMA 297, 1233-1240, https://doi.org/10.1001/jama.297.11.1233 (2007).

5. Topol, E. J. Failing the public health-rofecoxib, Merck, and the FDA. N Engl J Med 351, 1707-1709, https://doi.org/10.1056/ NEJMp048286 (2004).

6. Williams, G. Withdrawal of sibutramine in Europe. BMJ 340, c824, https://doi.org/10.1136/bmi.c824 (2010).

7. Hallas, J., Pottegard, A., Wang, S., Schneeweiss, S. \& Gagne, J. J. Persistent User Bias in Case-Crossover Studies in Pharmacoepidemiology. Am J Epidemiol, https://doi.org/10.1093/aje/kww079 (2016).

8. Rothman, K. J. Six persistent research misconceptions. J Gen Intern Med 29, 1060-1064, https://doi.org/10.1007/s11606-013-2755-z (2014).

9. Zhao, P. \& Bin, Y. On model selection consistency of Lasso. Journal of Machine learning research 7, 2541-2563 (2006).

10. Greenland, S. \& Robins, J. M. Empirical-Bayes adjustments for multiple comparisons are sometimes useful. Epidemiology 2, 244-251 (1991).

11. Mittleman, M. A. \& Mostofsky, E. Physical, psychological and chemical triggers of acute cardiovascular events: preventive strategies. Circulation 124, 346-354, https://doi.org/10.1161/CIRCULATIONAHA.110.968776 (2011). 
12. Mostofsky, E., Chahal, H. S., Mukamal, K. J., Rimm, E. B. \& Mittleman, M. A. Alcohol and Immediate Risk of Cardiovascular Events: A Systematic Review and Dose-Response Meta-Analysis. Circulation 133, 979-987, https://doi.org/10.1161/ CIRCULATIONAHA.115.019743 (2016).

13. Gurven, M. D. et al. Cardiovascular disease and type 2 diabetes in evolutionary perspective: a critical role for helminths? Evol Med Public Health, https://doi.org/10.1093/emph/eow028 (2016).

14. Pothineni, N. V. K. et al. Infections, atherosclerosis, and coronary heart disease. Eur Heart J 38, 3195-3201, https://doi.org/10.1093/ eurheartj/ehx362 (2017).

15. Horsdal, H. T., Sondergaard, F., Johnsen, S. P. \& Rungby, J. Antidiabetic treatments and risk of hospitalisation with myocardial infarction: a nationwide case-control study. Pharmacoepidemiol Drug Saf 20, 331-337, https://doi.org/10.1002/pds.2097 (2011).

16. Schlienger, R. G., Fischer, L. M., Jick, H. \& Meier, C. R. Current use of selective serotonin reuptake inhibitors and risk of acute myocardial infarction. Drug Saf 27, 1157-1165 (2004)

17. Noordam, R. et al. Use of antidepressants and the risk of myocardial infarction in middle-aged and older adults: a matched casecontrol study. Eur J Clin Pharmacol 72, 211-218, https://doi.org/10.1007/s00228-015-1972-2 (2016).

18. Singh, J. A., Ramachandaran, R., Yu, S. \& Curtis, J. R. Allopurinol use and the risk of acute cardiovascular events in patients with gout and diabetes. BMC Cardiovasc Disord 17, 76, https://doi.org/10.1186/s12872-017-0513-6 (2017).

19. Sauer, W. H., Berlin, J. A. \& Kimmel, S. E. Selective serotonin reuptake inhibitors and myocardial infarction. Circulation 104, 1894-1898 (2001).

20. Rajendra, N. S. et al. Mechanistic insights into the therapeutic use of high-dose allopurinol in angina pectoris. J Am Coll Cardiol 58, 820-828, https://doi.org/10.1016/j.jacc.2010.12.052 (2011).

21. Foretz, M., Guigas, B., Bertrand, L., Pollak, M. \& Viollet, B. Metformin: from mechanisms of action to therapies. Cell Metab 20, 953-966, https://doi.org/10.1016/i.cmet.2014.09.018 (2014).

22. Ryan, P. B., Madigan, D., Stang, P. E., Schuemie, M. J. \& Hripcsak, G. Medication-wide association studies. CPT Pharmacometrics Syst Pharmacol 2, e76, https://doi.org/10.1038/psp.2013.52 (2013).

23. Coloma, P. M. et al. Drug-induced acute myocardial infarction: identifying 'prime suspects' from electronic healthcare recordsbased surveillance system. PLoS One 8, e72148, https://doi.org/10.1371/journal.pone.0072148 (2013).

24. Funk, M. J. \& Landi, S. N. Misclassification in administrative claims data: quantifying the impact on treatment effect estimates. Curr Epidemiol Rep 1, 175-185, https://doi.org/10.1007/s40471-014-0027-z (2014).

25. Schneeweiss, S. et al. Real World Data in Adaptive Biomedical Innovation: A Framework for Generating Evidence Fit for DecisionMaking. Clin Pharmacol Ther 100, 633-646, https://doi.org/10.1002/cpt.512 (2016).

26. Ludvigsson, J. F. et al. External review and validation of the Swedish national inpatient register. BMC Public Health 11, 450, https:// doi.org/10.1186/1471-2458-11-450 (2011).

27. Govatsmark, R. E. S. et al. Completeness and correctness of acute myocardial infarction diagnoses in a medical quality register and an administrative health register. Scand J Public Health, 1403494818803256, https://doi.org/10.1177/1403494818803256 (2018).

28. Maclure, M. \& Mittleman, M. A. Should we use a case-crossover design? Annu Rev Public Health 21, 193-221, https://doi. org/10.1146/annurev.publhealth.21.1.193 (2000).

29. Klein, R. J. et al. Complement factor H polymorphism in age-related macular degeneration. Science 308, 385-389, https://doi. org/10.1126/science.1109557 (2005)

30. Yaspan, B. L. et al. Targeting factor D of the alternative complement pathway reduces geographic atrophy progression secondary to age-related macular degeneration. Sci Transl Med 9, https://doi.org/10.1126/scitranslmed.aaf1443 (2017).

31. Maclure, M. The case-crossover design: a method for studying transient effects on the risk of acute events. Am J Epidemiol 133, $144-153$ (1991).

32. Mittleman, M. A. et al. Triggering of acute myocardial infarction by heavy physical exertion. Protection against triggering by regular exertion. Determinants of Myocardial Infarction Onset Study Investigators. The New England journal of medicine 329, 1677-1683, https://doi.org/10.1056/NEJM199312023292301 (1993).

33. Mostofsky, E., Penner, E. A. \& Mittleman, M. A. Outbursts of anger as a trigger of acute cardiovascular events: a systematic review and meta-analysis. European heart journal 35, 1404-1410, https://doi.org/10.1093/eurheartj/ehu033 (2014).

34. Niederseer, D., Moller, J. \& Niebauer, J. Increased rates of myocardial infarction and deaths in men after sexual activity. International journal of cardiology 156, 234-235, https://doi.org/10.1016/j.ijcard.2012.01.036 (2012).

35. Brooke, H. L. et al. The Swedish cause of death register. Eur J Epidemiol 32, 765-773, https://doi.org/10.1007/s10654-017-0316-1 (2017).

36. Norwegian Cause of Death Registry. Available from, https://www.fhi.no/en/hn/health-registries/causeof-death-registry/. Last accessed 18 May (2017)

37. Kari, F. Establishment of the nationwide Norwegian Prescription Database (NorPD) - new opportunities for research in pharmacoepidemiology in Norway. Nor J Epidemiol 18, 129-136 (2008).

38. Wettermark, B. et al. The new Swedish Prescribed Drug Register-opportunities for pharmacoepidemiological research and experience from the first six months. Pharmacoepidemiol Drug Saf 16, 726-735, https://doi.org/10.1002/pds.1294 (2007).

39. Steenland, K., Bray, I., Greenland, S. \& Boffetta, P. Empirical Bayes adjustments for multiple results in hypothesis-generating or surveillance studies. Cancer Epidemiol Biomarkers Prev 9, 895-903 (2000).

40. Avalos, M. et al. Prescription-drug-related risk in driving: comparing conventional and lasso shrinkage logistic regressions. Epidemiology 23, 706-712, https://doi.org/10.1097/EDE.0b013e31825fa528 (2012).

41. Nee, M. et al. Prescription medicine use by pedestrians and the risk of injurious road traffic crashes: A case-crossover study. PLoS Med 14, e1002347, https://doi.org/10.1371/journal.pmed.1002347 (2017).

42. Bach, F. Bolasso: model consistent Lasso estimation through the bootstrap. Ithaca (New York): arXiv.org; 2008. arXiv.0804.1302, http://arxiv.org/abs/0804.1302. Accessed Jun 13 (2017).

43. Borenstein, M., Hedges, L. V., Higgins, J. P. \& Rothstein, H. R. A basic introduction to fixed-effect and random-effects models for meta-analysis. Res Synth Methods 1, 97-111, https://doi.org/10.1002/jrsm.12 (2010).

\section{Acknowledgements}

The authors would like to thank Dr. Svetlana Ondrasova Skurtveit, senior researcher at Norwegian Institute of Public Health for her valuable advices regarding the prescription databases and Professor Mette Langas at the Department of Mathematical Science, Norwegian University of Science and Technology for her important advices regarding LASSO. This research was supported by Helse Midt-Norge (grant number 46060913 to Dr. Janszky). The funding agency had no role in the study design, in the collection, analysis and interpretation of data, in the writing of the report, or in the decision to submit the article for publication. Dr. Sen received postdoctoral salary in the year 2014 to carry out his research from the Liaison committee between the Central Norway regional health Authority (Helse Midt-Norge) and The Norwegian University of Science and Technology. Dr. Brumpton works in a research unit funded by Stiftelsen Kristian Gerhard Jebsen; Faculty of Medicine and Health Sciences, NTNU; The Liaison Committee for education, research and innovation in Central Norway; and the Joint Research Committee between St. Olavs Hospital and the Faculty of Medicine and Health Sciences, NTNU; 
and the Medical Research Council Integrative Epidemiology Unit, University of Bristol which is supported by the Medical Research Council and the University of Bristol [MC_UU_12013/1]. Dr. Ljung is employed at the Swedish Medical Products Agency, SE-751 03 Uppsala, Sweden. The views expressed in this paper do not necessarily represent the views of the Government agency. Dr. Ljung has received consultancy fees from Pfizer in 2016, but not related to this project.

\section{Author Contributions}

A.S. has full access to all of the data in the study and takes responsibility for the integrity of the data and the accuracy of the data analysis. I.J., K.J.M., R.L., P.R.R. conceived and designed the study; I.J., R.L., I.J.B., P.R.R., B.M.B. acquired the data; A.S., I.V., B.H.L., B.M.B., L.B.S., I.J.B., L.J.V., P.R.R., R.L., K.J.M., I.J. analysed and interpreted the data; A.S., I.V., B.H.L., B.M.B., L.B.S., I.J.B., L.J.V., P.R.R., R.L., K.J.M., I.J. critically revised the manuscript for important intellectual content. A.S., K.J.M., I.J. drafted the manuscript. I.J.B., B.M.B., R.L. provided administrative, technical or logistic support. A.S., R.L., I.V., B.M.B. carried out the statistical analysis. I.J. obtained funding. I.J., K.L.M., R.L. supervised the study. A.S. and I.J. are the guarantors of Norwegian data and R.L. is the guarantor of Swedish data.

\section{Additional Information}

Supplementary information accompanies this paper at https://doi.org/10.1038/s41598-019-44641-1.

Competing Interests: The authors declare no competing interests.

Publisher's note: Springer Nature remains neutral with regard to jurisdictional claims in published maps and institutional affiliations.

(c) (i) Open Access This article is licensed under a Creative Commons Attribution 4.0 International License, which permits use, sharing, adaptation, distribution and reproduction in any medium or format, as long as you give appropriate credit to the original author(s) and the source, provide a link to the Creative Commons license, and indicate if changes were made. The images or other third party material in this article are included in the article's Creative Commons license, unless indicated otherwise in a credit line to the material. If material is not included in the article's Creative Commons license and your intended use is not permitted by statutory regulation or exceeds the permitted use, you will need to obtain permission directly from the copyright holder. To view a copy of this license, visit http://creativecommons.org/licenses/by/4.0/.

(c) The Author(s) 2019 